\title{
Patient Report
}

\section{Young athlete with sudden cardiac arrest treated with therapeutic hypothermia}

\author{
Katsuaki Kojima, ${ }^{1 \dagger}$ Akihiro Sato, ${ }^{1}$ Yukio Yamashita, ${ }^{1}$ Jun Ishihara, ${ }^{1}$ Hideshi Aoyagi ${ }^{2}$ and Kaoru Okishige ${ }^{2}$ \\ ${ }^{1}$ Department of Pediatrics, Yokohama Municipal Citizen's Hospital and ${ }^{2}$ Heart Center, Yokohama-City Bay Red Cross \\ Hospital, Yokohama, Kanagawa, Japan
}

\begin{abstract}
Reported herein is a coronary anomaly that occurred in a young adolescent athlete who presented with cardiopulmonary arrest. The patient was resuscitated and treated with therapeutic hypothermia. The patient had no associated neurological complications at follow up. Enhanced computed tomography of the heart indicated an anomalous left main coronary artery originating from the right coronary sinus and coursing between the aorta and the pulmonary artery. The patient underwent surgical intervention with coronary artery bypass grafting to prevent symptom recurrence.
\end{abstract}

Key words cardiac arrest, coronary artery anomaly, therapeutic hypothermia.

The anomalous origin of the left main coronary artery (LCA) from the right coronary sinus is a rare anomaly and may lead to sudden cardiac death (SCD) in otherwise healthy children during exercise.

Therapeutic hypothermia has been shown to improve neurological outcome and decrease mortality in adults who experience cardiac arrest in an out-of-hospital setting. In children and adolescents, however, evidence regarding the effectiveness of this method is very limited. Here, we describe a pediatric case of cardiopulmonary arrest (CPA), resuscitation, and subsequent treatment with therapeutic hypothermia in an adolescent with congenital coronary artery anomaly.

\section{Case report}

A 13-year-old boy with no prior history of illness suffered CPA while playing soccer at school. His previous electrocardiogram at age 12 showed no abnormality. He had no prodromal symptoms before the event. His coach immediately initiated cardiopulmonary resuscitation (CPR). Emergency medical services (EMS) arrived at the scene $10 \mathrm{~min}$ after the boy collapsed and confirmed cardiac arrest. EMS personnel continued CPR with bag-mask ventilation and chest compression. The patient was defibrillated twice with an automated external defibrillator (AED) by the EMS personnel en route to the hospital. Ventricular fibrillation was the initial recorded rhythm in the patient. Sinus tachycardia with

Correspondence: Katsuaki Kojima, MD, Department of Pediatrics, Yokohama Municipal Citizen's Hospital, Yokohama, Kanagawa 2408555, Japan. Email: fi990071@ gmail.com

†Present address: Department of Pediatrics, Children's Hospital of Michigan, Wayne State University, 3901 Beaubien Street, Detroit, MI 48201, USA.

Received 16 December 2012; revised 26 February 2013; accepted 16 May 2013. sustained circulation was achieved after second rescue shock. A review of the patient's prehospital records showed that he returned to spontaneous circulation $23 \mathrm{~min}$ after the collapse.

Upon arrival at the emergency department, the patient's blood pressure was $72 / 54 \mathrm{mmHg}$, pulse was 155 beats $/ \mathrm{min}$, and temperature was $37.2^{\circ} \mathrm{C}$ via a rectal thermometer. Initial neurological examination in the emergency department indicated coma (Glasgow coma scale score, 3), sluggish pupillary light reflex, and diminished respiratory effort. Cardiovascular examination was normal. The patient was intubated immediately upon arrival at the emergency department. Laboratory results were as follows: serum aspartate aminotransferase, $253 \mathrm{U} / \mathrm{L}$; alanine aminotransferase, $356 \mathrm{U} / \mathrm{L}$; lactate dehydrogenase, $604 \mathrm{U} / \mathrm{L}$; creatine phosphokinase, $4382 \mathrm{U} / \mathrm{L}$ with myocardial bands of $554 \mathrm{U} / \mathrm{L}$; troponin I, $3.50 \mathrm{ng} / \mathrm{mL}$; and lactate, $108.9 \mathrm{mg} / \mathrm{dL}$. His initial serum glucose level was $200 \mathrm{mg} / \mathrm{dL}$. Chest radiograph showed no congestion, and the cardiothoracic ratio was $54 \%$. The results of a 12-lead electrocardiogram indicated sinus tachycardia with non-specific ST changes. Bedside emergency echocardiogram demonstrated normal ventricular function with no effusion, ventricular hypertrophy, or organic heart disease. Non-contrast computed tomography (CT) of the brain was unremarkable. After discussion of the risks and benefits of treatment with therapeutic hypothermia, the family agreed to begin therapy.

Ice packs and a surface cooling system with sedative measures and infusion of neuromuscular blockers were used to induce hypothermia. I.v. insulin infusion was also initiated. A target temperature of $34^{\circ} \mathrm{C}$ was reached approximately $5 \mathrm{~h}$ after the collapse. Rectal temperature was maintained between $33^{\circ} \mathrm{C}$ and $34^{\circ} \mathrm{C}$ for the next $24 \mathrm{~h}$. The patient required no vasopressors during the hospital course. On day 1, verapamil hydrochloride was infused for treating paroxysmal supraventricular tachycardia. Over the next $48 \mathrm{~h}$, the patient was slowly rewarmed until his temperature reached $36^{\circ} \mathrm{C}$, upon which sedation measures and neuromuscular blockers were discontinued. Subsequently, the 
patient responded to pain. On day 5, he was extubated. Physical examination at this time indicated no neurological abnormalities. Electroencephalography and magnetic resonance imaging of the brain indicated no abnormal findings. Cerebral Performance Category (CPC) score at 1 month and at 3 months after the event were both CPC 1 .

Enhanced CT confirmed the course of the anomalous LCA originating with a separate ostium from the right coronary sinus (Fig. 1). The LCA subsequently coursed between the aorta and the pulmonary artery. Enhanced CT also showed no evidence of atherosclerotic coronary artery disease. To reduce the risk of future episodes of myocardial ischemia and SCD, surgical intervention with coronary artery bypass grafting was performed. The left internal mammary artery was anastomosed to the left anterior descending artery. The LCA was ligated at the left main trunk to avoid competitive flow. No coronary spasm or arrhythmia was noted at 6 months after the surgery.

\section{Discussion}

We have described the case of a young adolescent athlete with CPA, who was resuscitated and subsequently treated with therapeutic hypothermia. The patient had no neurological complications at follow up. Enhanced CT confirmed the diagnosis of anomalous origin of the LCA.

In spite of the relative success of the initial resuscitation efforts in cases of pediatric cardiac arrest $(>60 \%$ of victims are revived with the restoration of circulation), the 1 year survival rate of children following cardiac arrest remains poor $(<35 \%) .{ }^{1}$ Hypoxicischemic encephalopathy is the most frequent cause of morbidity and mortality following resuscitation, and severity is affected by the duration of cardiac arrest as well as the effectiveness of the resuscitation effort. AED has been reported to contribute to both

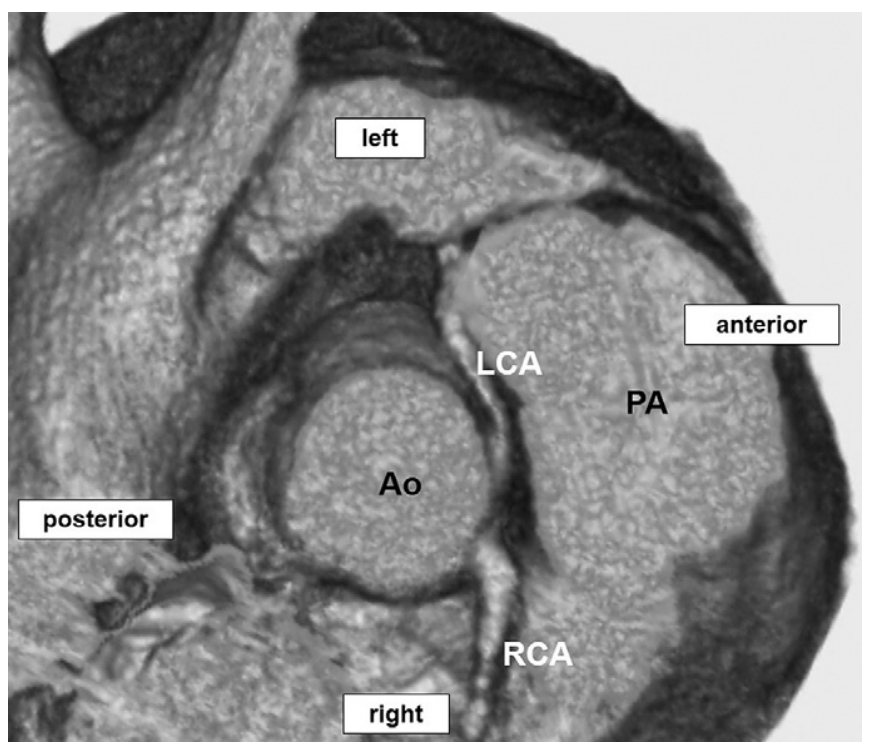

Fig. 1 Reconstructed 3-D computed tomography: anomalous left main coronary artery (LCA) arising from the right coronary sinus with an acute angle of origin and coursing between the aorta (Ao) and pulmonary artery (PA). RCA, right coronary artery. improved survival and reduced neurological complications when used in patients with sudden cardiac arrest. Recently, a nationwide dissemination of AED in public places throughout Japan has been reported to have improved the survival rate among patients with out-of-hospital cardiac arrest. ${ }^{2}$ The present patient received immediate resuscitation with an AED performed by EMS followed by subsequent inpatient treatment, and the patient had no evidence of neurological complications at follow up.

The American Heart Association and the International Liaison Committee on Resuscitation recommend that therapeutic hypothermia $\left(32-34^{\circ} \mathrm{C}\right.$ for $\left.12-24 \mathrm{~h}\right)$ should be considered if a child or adolescent remains comatose after resuscitation. ${ }^{3}$ Evidence supporting the effectiveness of therapeutic hypothermia in children, however, remains limited; ideal methods for cooling and rewarming the patient, as well as the optimal duration of therapy, are unknown. ${ }^{3}$ For example, in one retrospective study of 222 pediatric patients with cardiac arrest, evidence supporting the effectiveness of hypothermia therapy was inconclusive, but the authors of that retrospective study suggested using guidelines for hypothermia therapy and rewarming techniques in future studies. ${ }^{4}$

Even though the optimal rate for inducing therapeutic hypothermia and the ideal duration of treatment are unknown, both human and animal studies have provided a wealth of evidence in support of this treatment measure. The target temperature of $32-34^{\circ} \mathrm{C}$ should be achieved within $6 \mathrm{~h}^{5}$ and maintained for 12-24 h. ${ }^{6}$ Because rapid rewarming can cause electrolyte abnormalities, cerebral edema, seizures, and other problems, a rewarming rate of $<0.2-0.25^{\circ} \mathrm{C}$ is generally recommended.

Sudden cardiac death associated with athletic activity is a rare but devastating event. The incidence of SCD among competitive athletes is considerably low; it is estimated to be between 1 per 50000 and 1 per 300000 athletes in a 10-20 year period. ${ }^{7}$ Congenital coronary artery anomalies are reported to be the second most frequent cause (17\%) of SCD in young competitive athletes. $^{8}$

Obtaining an accurate diagnosis in the case of a coronary anomaly is challenging especially in asymptomatic patients because physical examination is often unrevealing. When a coronary anomaly is suspected, echocardiography may establish the diagnosis, but false negatives can occur in this method. Of 12 athletes who had sudden death due to coronary anomaly, echocardiographic evaluation of two patients produced a false-negative diagnosis. ${ }^{9}$ In contrast, non-invasive coronary magnetic resonance angiography and coronary CT angiography (CCTA) are increasingly being used to define anomalous coronary anatomy because they are more capable of delineating the anatomical relationship between the coronary artery and the surrounding structures. ${ }^{10}$ In the present case, CTTA accurately identified the coronary anomaly after an unremarkable echocardiogram.

The anatomical course of an anomalous coronary artery is very important in determining prognosis and treatment. An arterial course between the aorta and pulmonary artery, as in the present case, has been reported to be associated with poor prognosis, because the anomalous course may cause compression of the vessel, myocardial ischemia, or even sudden death. CCTA has an advantage and is preferred over coronary angiography because 
it is able to identify the course of the coronary artery. The precise course of the anomalous vessel may be difficult to delineate on coronary angiography because of its complex 3-D geometry displayed fluoroscopically in two dimensions. In contrast, in the present case, CCTA enabled a clear depiction of the course of the anomalous LCA, as well as the relation of the anomalous vessel to the surrounding structures.

\section{Conclusion}

This case illustrates how a congenital coronary artery anomaly can manifest in children and adolescents with sequelae that include CPA. Good neurological outcome may be achieved with immediate resuscitation efforts as well as therapeutic hypothermia.

\section{References}

1 Schindler MB, Bohn D, Cox PN et al. Outcome of out-of-hospital cardiac or respiratory arrest in children. N. Engl. J. Med. 1996; 335: 1473-9.

2 Kitamura T, Iwami T, Kawamura $\mathrm{T}$ et al. Nationwide publicaccess defibrillation in Japan. N. Engl. J. Med. 2010; 362: 994 1004
3 Kleinman ME, Chameides L, Schexnayder SM et al. Pediatric advanced life support: 2010 American Heart Association Guidelines for Cardiopulmonary Resuscitation and Emergency Cardiovascular Care. Pediatrics 2010; 126: e1361-99.

4 Doherty DR, Parshuram CS, Gaboury I et al. Hypothermia therapy after pediatric cardiac arrest. Circulation 2009; 119: 1492-500.

5 Nielsen N, Hovdenes J, Nilsson F et al. Outcome, timing and adverse events in therapeutic hypothermia after out-of-hospital cardiac arrest. Acta Anaesthesiol. Scand. 2009; 53: 926-34.

6 Hypothermia after Cardiac Arrest Study G. Mild therapeutic hypothermia to improve the neurologic outcome after cardiac arrest. N. Engl. J. Med. 2002; 346: 549-56.

7 Maron BJ, Shirani J, Poliac LC, Mathenge R, Roberts WC, Mueller FO. Sudden death in young competitive athletes. Clinical, demographic, and pathological profiles. JAMA 1996; 276: 199-204.

8 Maron BJ, Doerer JJ, Haas TS, Tierney DM, Mueller FO. Sudden deaths in young competitive athletes: Analysis of 1866 deaths in the United States, 1980-2006. Circulation 2009; 119: 1085-92.

9 Basso C, Maron BJ, Corrado D, Thiene G. Clinical profile of congenital coronary artery anomalies with origin from the wrong aortic sinus leading to sudden death in young competitive athletes. J. Am. Coll. Cardiol. 2000; 35: 1493-501.

10 Cademartiri F, La Grutta L, Malago R et al. Prevalence of anatomical variants and coronary anomalies in 543 consecutive patients studied with 64-slice CT coronary angiography. Eur. Radiol. 2008; 18: 781-91. 